\title{
In the news
}

\section{AAIC 2016 LIGHTS UP TORONTO}

The 2016 Alzheimer's Association International Conference (AAIC 16), which was held in Toronto, Canada (24-28 July 2016), attracted over 5,000 delegates, with interests ranging from molecular and cell biology to psychosocial and environmental issues in Alzheimer disease (AD). On the first night of the conference, the $\mathrm{CN}$ Tower was illuminated in purple - the colour of the Alzheimer's Association - to mark the event.

A number of newsworthy findings were reported at AAIC 2016. New health administrative data from Ontario indicated a rise of almost $20 \%$ in the prevalence of $A D$ and dementia in this Canadian province over the past 8 years. The increase was especially marked in men, although women continued to carry the bulk of the dementia burden in this region.

In a similar vein, a report from the Mayo Clinic (Jacksonville, Florida) suggested that dementia is misdiagnosed more often in men than in women. According to Melissa Murray, who presented the work, "atypical clinical presentations were more common in men, suggesting that their lower reported prevalence of $A D$ may be a result of the disease not being accurately recognized in life."

The contribution of lifestyle factors to AD risk and prevention was high on the agenda at AAIC 2016. In a plenary talk, Laura Baker from Wake Forest School of Medicine highlighted the potential role of 'exercise as medicine' in people with cognitive impairment. In addition, data from the University of Wisconsin indicated that complex occupations involving interactions with people were associated with increased levels of cognitive reserve, as indicated by the ability to maintain cognitive function despite accumulation of white matter pathology in the brain.

Basic science was a particular focus of the conference. A new series, entitled Emerging Concepts in Basic Science, included sessions on secretase biology, the yin and yang of TREM2, and tau in AD, and culminated in a panel discussion involving the session chairs.

New sources of AD biomarkers were also a hot topic at AAIC 2016. Studies presented at the conference suggested that retinal thinning and olfactory dysfunction are early clinical features of $A D$, and might aid detection and management of the condition.

"It is the goal of the Alzheimer's Association and AAIC - the world's largest forum for the dementia research community - to report on and reflect the state of the art in all aspects of AD and dementia research," concluded Maria Carrillo, Chief Science Officer of the Alzheimer's Association. "Research represents hope, and it is our goal to lead the way to a world without AD." 